Michą BARAN (Warszawa)

\title{
QUANTILE HEDGING ON MARKETS WITH PROPORTIONAL TRANSACTION COSTS
}

Abstract. The problem of risk measures in a discrete-time market model with transaction costs is studied. Strategy effectiveness and shortfall risk are introduced. This gives a generalization of quantile hedging presented in [4].

1. Introduction. It is well known that on a classical market without transaction costs the price $x_{0}$ of a contingent claim $C$ is given as $x_{0}=\sup _{Q \in \mathcal{Q}} \mathbf{E}^{Q}[C]$, where $\mathcal{Q}$ is the set of all martingale measures equivalent to the objective measure $P$. This means that if we have an initial endowment $x \geq x_{0}$ then we can hedge $C$. Thus for $x$ there exists a self-financing strategy $B$ for which the terminal value $X_{T}^{x, B}$ is not smaller than $C$. If $x<x_{0}$ then we no longer can hedge $C$ : for each strategy $B$ we have $P\left(X_{T}^{x, B}<C\right)>0$. The investor who wants to hedge $C$ in some way must consider some risk connected with the fact that he is not able to hedge $C$ entirely. Many risk measures have been introduced, for instance by Cvitanić and Karatzas [1], Pham [8], and Föllmer and Leukert [4], [5]. Cvitanić and Karatzas [1] study the risk measure $\inf _{B \in \mathcal{B}} \mathbf{E}\left[\left(C-X_{T}^{x, B}\right)^{+}\right]$, where $\mathcal{B}$ is the set of all self-financing strategies. Pham $[8]$ introduces $L^{p}$ hedging and his risk measure is defined as $\inf _{B \in \mathcal{B}} \mathbf{E}\left[l_{p}\left(\left(C-X_{T}^{x, B}\right)^{+}\right)\right]$, where $l_{p}(x)=x^{p} / p$. Other examples of risk measures are provided by Föllmer and Leukert [4]. They consider the so called quantile hedging problem and define a random variable connected with the strategy $(x, B)$ by

$$
\varphi_{x, B}=\mathbf{1}_{\left\{X_{T}^{x, B} \geq C\right\}}+\frac{X_{T}^{x, B}}{C} \mathbf{1}_{\left\{X_{T}^{x, B}<C\right\}} .
$$

2000 Mathematics Subject Classification: 60G42, 91B28, 91B24, 91B30.

GEL Classification Numbers: G11, G13.

Key words and phrases: quantile hedging, shortfall risk, transaction costs, risk measures. 
It is called the "success function" and its expectation is an effectiveness measure connected with the strategy $(x, B)$. The success function takes its values in the interval $[0,1]$. If $(x, B)$ is a hedging strategy, then $\varphi_{x, B}=1$, otherwise $P\left(\varphi_{x, B}<1\right)>0$, which implies $\mathbf{E}\left[\varphi_{x, B}\right]<1$. Föllmer and Leukert's aim is to find a strategy $B$ maximizing $\mathbf{E}\left[\varphi_{x, B}\right]$ for a given $x$. In [5] they also examine another risk measure, $\inf _{B \in \mathcal{B}} \mathbf{E}\left[l\left(\left(C-X_{T}^{x, B}\right)^{+}\right)\right]$, where $l$ is a loss function.

In this paper we study the problem of risk measures on markets with proportional transaction costs. The main idea is based on the papers of Föllmer and Leukert on quantile hedging [4] and minimizing shortfall risk [5]. On markets with transaction costs we are given a multi-dimensional contingent claim $H$, a multi-dimensional wealth process $V_{t}^{v, B}$, and some cone $K_{T}$ which is constructed on the basis of transaction costs. The cone $K_{T}$ determines a partial ordering " $\succeq_{T}$ " in $\mathbb{R}^{d}$ such that $x \succeq_{T} y \Leftrightarrow x-y \in K_{T}$. We say that a strategy $(v, B)$ hedges $H$ if $V_{T}^{v, B} \succeq_{T} H$. Papers [2], [6] and [7] provide a characterization of the set $\Gamma(H) \subseteq R^{d}$ of initial endowments $v$ for which there exists a hedging strategy $B$ such that $V_{T}^{v, B} \succeq_{T} H$. The problem arises what is an optimal strategy for an initial endowment $v \notin \Gamma(H)$. For the terminal wealth $V_{T}^{v, B}$ we introduce a set $\mathfrak{L}\left(V_{T}^{v, B}, H\right)$ of proportional transfers. Simply speaking, for $L \in \mathfrak{L}\left(V_{T}^{v, B}, H\right)$ we have

$$
\frac{\left(V_{T}^{v, B} \mid L\right)^{i}}{H^{i}}=\frac{\left(V_{T}^{v, B} \mid L\right)^{j}}{H^{j}}
$$

for all $i, j$ where $V_{T}^{v, B} \mid L$ is the terminal wealth after the proportional transfer $L$. For this ratio we write

$$
\frac{V_{T}^{v, B} \mid L}{H}:=\frac{\left(V_{T}^{v, B} \mid L\right)^{i}}{H^{i}} .
$$

In Section 3 we introduce the "success function" whose expectation is an effectiveness measure of the strategy $(v, B)$, by setting

$$
\varphi_{v, B}=\mathbf{1}_{\left\{V_{T}^{v, B} \succeq_{T} H\right\}}+\operatorname{esssup}_{L \in \mathfrak{L}\left(V_{T}^{v, B}, H\right)} \frac{V_{T}^{v, B} \mid L}{H} \mathbf{1}_{\left\{V_{T}^{v, B} \succeq_{T} H\right\}^{c}} .
$$

We establish some useful properties of the success function. It appears that $\varphi_{v, B} \in[0,1]$ and if $v \in \Gamma(H)$ then $\varphi_{v, B}=1$ for the hedging strategy $B$, whereas for $v \notin \Gamma(H)$ we have $P\left(V_{T}^{v, B}<1\right)>0$ for each strategy $B$, which implies $\mathbf{E}\left[\varphi_{v, B}\right]<1$. Our aim is to find, for the initial endowment $v$, a strategy $B$ maximizing $\mathbf{E}\left[\varphi_{v, B}\right]$. We also consider another problem. For $1 \geq \varepsilon \geq 0$ we characterize the set $\Gamma_{\varepsilon}(H) \subseteq \mathbb{R}^{d}$ of initial endowments for which there exists a strategy $B$ such that $\mathbf{E}\left[\varphi_{v, B}\right] \geq 1-\varepsilon$. These two aspects of quantile hedging are analogous to the problems considered by Föllmer and Leukert. 
In Section 6, we introduce a shortfall risk in quantile hedging. The shortfall is defined as

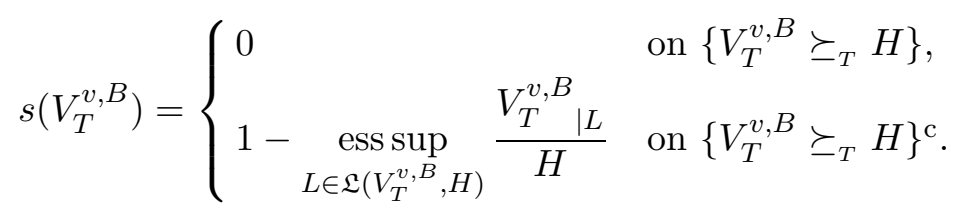

It is a $[0,1]$-valued random variable which is equal to 0 if $V_{T}^{v, B} \succeq_{T} H$ and is strictly positive otherwise. It describes the part of the contingent claim which is not hedged by the strategy $(v, B)$. We study the problem of minimizing the shortfall risk given as $\mathbf{E}\left[u\left(s\left(V_{T}^{v, B}\right)\right)\right]$, where $u:[0,1] \rightarrow \mathbb{R}$ is a loss function. We make the assumption that the investor considers as a loss only the percentage of the contingent claim which is not hedged, and not the value of this part. As before, we study two problems. First, in Section 6, we characterize the strategy $B$ which minimizes the shortfall risk. Next, in Section 7 , we characterize the set $\Gamma_{\alpha}^{u}(H)$ of initial endowments for which there exists a strategy $B$ such that $\mathbf{E}\left[u\left(s\left(V_{T}^{v, B}\right)\right)\right] \leq \alpha$ for a given $\alpha \geq 0$.

In Section 8 we show how Föllmer and Leukert's theory can be obtained under zero transaction costs. Since the condition EF imposed in [6] is not satisfied, we use the results of [2].

2. Market with proportional transaction costs. In this section we present some results obtained by Kabanov, Rásonyi, and Stricker in [6] and [7], which deal with conditions for the absence of arbitrage under friction. In particular, we need a hedging theorem providing a description of the set of initial endowments which allow hedging the contingent claim.

Let $\left(\Omega, \mathcal{F},\left(\mathcal{F}_{t}\right)_{t=0,1, \ldots, T}, P\right)$ be a probability space equipped with a complete, discrete-time filtration. We assume that $\mathcal{F}_{0}$ is the trivial $\sigma$-field and that $\mathcal{F}_{T}=\mathcal{F}$. On $\Omega$ we are given a strictly positive $\mathbb{R}^{d}$-valued adapted process $S_{t}$ which describes the prices of $d$ traded securities. We can assume that, for instance, the first component is the price of a bond, but this is not necessary for further considerations. Proportional transaction costs are given as the process $\Lambda_{t}=\left(\lambda_{t}^{i, j}\right)_{i, j=1, \ldots, d}$ with values in the set $M_{+}^{d}$ of matrices with non-negative, adapted entries and zero diagonal. If we want to increase the $j$ th stock account by an amount $L^{i j} \geq 0$ at time $t$, then we have to transfer the amount $\left(1+\lambda_{t}^{i j}\right) L^{i j}$ from the $i$ th account. The quantity $\lambda_{t}^{i j} L^{i j}$ is lost because of the transaction costs involved. Given an initial endowment $v \in \mathbb{R}^{d}$ we invest in stocks at each time $t=0,1, \ldots, T$. The agent's position at time $t$ can be described either by a vector $\widehat{V}_{t}$ of stock units or by a vector $V_{t}$ of values invested in each stock. The relation between these quantities is: $V_{t}^{i}=\widehat{V}_{t}^{i} S_{t}^{i}$. The operator "^" will also be used for any random vector $Z$ : then $\widehat{Z}$ stands for $\left(Z^{1} / S^{1}, \ldots, Z^{d} / S^{d}\right)$. A self-financing portfolio is defined 
by its increments as follows:

$$
\Delta V_{t}^{i}=\widehat{V}_{t-1}^{i} \cdot \Delta S_{t}^{i}+\Delta B_{t}^{i}, \quad i=1, \ldots, d, t=0,1, \ldots, T,
$$

with initial values $V_{-1}^{i}=v^{i}, S_{-1}=S_{0}, L_{-1}^{i j}=0$ for $i, j=1, \ldots, d$, and where

$$
\Delta B_{t}^{i}:=\sum_{j=1}^{d} \Delta L_{t}^{j i}-\sum_{j=1}^{d}\left(1+\lambda_{t}^{i j}\right) \Delta L_{t}^{i j} .
$$

Here $\Delta Y_{t}=Y_{t}-Y_{t-1}$ for each process $Y$. The adapted, increasing and nonnegative process $L^{i j}$ represents the net cumulative transfers from position $i$ to position $j$ under transaction costs. The increment $\Delta V_{t}^{i}$ of the value on the $i$ th stock account consists of two parts: the increment $\widehat{V}_{t}^{i} \Delta S_{t}^{i}$ due to price movements and the increment $\Delta B_{t}^{i}$ caused by the agent's action at time $t$. Since the pair $(v, B)$ determines the wealth process $V_{t}^{v, B}$, we will treat it as a trading strategy.

For $A \subseteq \mathbb{R}^{d}$, we denote by $L^{0}\left(A, \mathcal{F}_{t}\right)$ the set of $\mathcal{F}_{t}$-measurable random variables with values in $A ; L^{0}\left(M_{+}^{d}, \mathcal{F}_{t}\right)$ stands for the matrices whose entries are non-negative, $\mathcal{F}_{t}$-measurable random variables. Let

$$
\begin{aligned}
& M_{t}(\omega):=\left\{x \in \mathbb{R}^{d}: \text { there exists } L \in L^{0}\left(M_{+}^{d}, \mathcal{F}_{t}\right)\right. \text { such that } \\
& \left.\qquad x^{i}=\sum_{j=1}^{d}\left(1+\lambda_{t}^{i j}(\omega)\right) L^{i j}(\omega)-\sum_{j=1}^{d} L^{j i}(\omega)\right\}
\end{aligned}
$$

be the set of positions which can be converted into zero by a non-negative transfer. This set is a polyhedral cone. Let $K_{t}:=\mathbb{R}_{+}^{d}+M_{t}$ and $F_{t}:=$ $K_{t} \cap\left(-K_{t}\right)$. The set $K_{t}$, which is called the solvency region, is a polyhedral cone. It is formed by the vectors which can be transformed into a vector with only non-negative components by a positive transfer, that is, by adding a vector from $-M_{t}$. The set $F_{t}$ represents the positions which can be converted into zero and vice versa; it is a linear space.

We shall say that a strategy $(0, B)$ is a weak arbitrage opportunity at time $t$ if $V_{t}^{0, B} \in K_{t}$ and $P\left(V_{t}^{0, B} \in K_{t} \backslash F_{t}\right)>0$. Absence of weak arbitrage opportunities means that there is no arbitrage opportunity at any time. The absence of weak arbitrage opportunities (strict no-arbitrage property) can be expressed in geometric terms:

$$
\mathbf{N A}^{\mathrm{s}}: \quad R_{t} \cap L^{0}\left(K_{t}, \mathcal{F}_{t}\right) \subseteq L^{0}\left(F_{t}, \mathcal{F}_{t}\right) \quad \text { for } t=0,1, \ldots, T,
$$

where

$$
R_{t}:=\left\{V_{t}^{0, B}: B \in \mathcal{B}\right\}
$$

and $\mathcal{B}$ is the set of all strategies. The set $R_{t}$ describes the wealth at time $t$ which can be obtained by starting with the zero initial endowment. 
Let us define an efficient friction condition:

EF : $\quad$ The cones $K_{t}(\omega)$ are proper, i.e. $F_{t}(\omega)=\{0\}$ for each $(\omega, t)$.

Under $\mathbf{E F}$ the condition $\mathbf{N A}^{\mathrm{s}}$ can be rewritten as $R_{t} \cap L^{0}\left(K_{t}, \mathcal{F}_{t}\right)=\{0\}$ for $t=0,1, \ldots, T$. Under $\mathbf{E F}$ there are some conditions equivalent to $\mathbf{N} \mathbf{A}^{\mathrm{s}}$. For more details see [6].

The most important tool for this paper is a description of the set of initial endowments which allow one to hedge the contingent claim. Let us start with the fact that the cone $K_{t}$ generates a partial ordering " $\succeq_{t}$ " on $\mathbb{R}^{d}$ such that $x \succeq_{t} y \Leftrightarrow x-y \in K_{t}$. A contingent claim $H$ is an $\mathbb{R}^{d}$-valued random variable, and

$$
\Gamma(H):=\left\{v \in \mathbb{R}^{d} \text { : there exists a strategy } B \text { such that } V_{T}^{v, B} \succeq_{T} H\right\}
$$

stands for all hedging initial endowments. For simplicity we assume that $H \succeq_{T} c \mathbf{1}$ for some $c \in \mathbb{R}$. The next theorem, given in [7], provides a description of the set $\Gamma(H)$.

Theorem 2.1. Assume that $\mathbf{E F}$ and $\mathbf{N A}^{\mathrm{s}}$ are satisfied. Then

$$
\Gamma(H)=\left\{v \in \mathbb{R}^{d}: \widehat{Z}_{0} v \geq \mathbf{E} \widehat{Z}_{T} H \forall Z \in \mathcal{Z}\right\},
$$

where $\mathcal{Z}$ is the set of bounded martingales such that $\widehat{Z}_{t} \in L^{0}\left(K_{t}^{*}, \mathcal{F}_{t}\right)$ for $t=0,1, \ldots, T$ and $K_{t}^{*}$ denotes the dual cone to $K_{t}$.

From now on we assume that the conditions $\mathbf{E F}$ and $\mathbf{N A} \mathbf{A}^{\mathrm{s}}$ are satisfied.

3. Strategy effectiveness. In this section we introduce a success function $\varphi_{v, B}$ for the strategy $(v, B)$ and establish its properties. Its expectation under $P$ is in fact some kind of risk measure, but a more adequate risk measure will be defined in Section 6. The expectation appearing here will rather be taken as an effectiveness measure.

We will consider only admissible strategies and from now on we assume that $H \succeq_{T} 0$ almost everywhere.

Definition 3.1. A strategy $(v, B)$ is admissible if $V_{T}^{v, B} \succeq_{T} 0$.

Let $(v, B)$ be an admissible strategy. Our aim is to describe its effectiveness regarding the contingent claim $H$. Divide $\Omega$ into two parts: $\left\{V_{T}^{v, B} \succeq_{T} H\right\}$ and $\left\{V_{T}^{v, B} \succeq_{T} H\right\}^{\mathrm{c}}$. On $\left\{V_{T}^{v, B} \succeq_{T} H\right\}$ we put $\varphi_{v, B}=1$. The next part of this section is denoted to defining $\varphi_{v, B}$ on $\left\{V_{T}^{v, B} \succeq_{T} H\right\}^{\mathrm{c}}$ and examining its basic properties.

For the terminal wealth $V_{T}^{v, B}$ and transfer $L \in L^{0}\left(M_{+}^{d}, \mathcal{F}_{T}\right)$ we will consider $V_{T}^{v, B}$ after transfer $L$ under transaction costs at time $T$, given by

$$
\left(V_{T}^{v, B} \mid{ }^{i}\right)^{i}=\left(V_{T}^{v, B}\right)^{i}+\sum_{j=1}^{d} L^{j i}-\sum_{j=1}^{d}\left(1+\lambda_{T}^{i j}\right) L^{i j} .
$$


In the set $L^{0}\left(M_{+}^{d}, \mathcal{F}_{T}\right)$ of all transfers we distinguish a subclass of proportional transfers.

Definition 3.2. Assume that $V_{T}^{v, B} \nsucceq_{T} H$ for some admissible strategy $(v, B)$. A transfer $L \in L^{0}\left(M_{+}^{d}, \mathcal{F}_{T}\right)$ is proportional if there exists $c_{L} \in$ $L^{0}\left(\mathbb{R}, \mathcal{F}_{T}\right)$ such that

$$
V_{T}^{v, B}{ }_{\mid L}=c_{L} \cdot H
$$

$\mathfrak{L}\left(V_{T}^{v, B}, H\right)$ stands for the class of all proportional transfers, and for $L \in$ $\mathfrak{L}\left(V_{T}^{v, B}, H\right)$ we write $V_{T}^{v, B} \mid L=: c_{L}$.

REMARK 3.3. $\mathfrak{L}\left(V_{T}^{v, B}, H\right)$ is not empty since $(v, B)$ is admissible. This means that there exists $L_{0} \in L^{0}\left(M_{+}^{d}, \mathcal{F}_{T}\right)$ for which $V_{T}^{v, B} \mid L_{0}=0$, and thus $c_{L_{0}}=V_{T}^{v, B}{ }_{\mid L_{0}} / H=0$.

The meaning of the class of proportional transfers is to achieve the same "rate of hedging" on each stock account. We want to make this rate as high as possible. Therefore on the set $\left\{V_{T}^{v, B} \succeq_{T} H\right\}^{\mathrm{c}}$ we define $\varphi_{v, B}$ as

$$
\operatorname{ess}_{L \in \mathfrak{L}\left(V_{T}^{v, B}, H\right)} \frac{V_{T}^{v, B} \mid L}{H} .
$$

This leads to the following definition of the success function:

$$
\varphi_{v, B}=\mathbf{1}_{\left\{V_{T}^{v, B} \succeq_{T} H\right\}}+{\operatorname{ess} \sup _{L \in \mathfrak{L}\left(V_{T}^{v, B}, H\right)}}_{\frac{V_{T}^{v, B} \mid L}{H}} \mathbf{1}_{\left\{V_{T}^{v, B} \succeq_{T} H\right\}^{\mathrm{c}}} .
$$

Lemma 3.4. Assume that $V_{T}^{v, B} \nsucceq_{T} H$. Then there exists an optimal transfer $\widehat{L} \in \mathfrak{L}\left(V_{T}^{v, B}, H\right)$ such that

$$
\operatorname{esssup}_{L \in \mathfrak{L}\left(V_{T}^{v, B}, H\right)} \frac{V_{T}^{v, B} \mid L}{H}=\frac{V_{T}^{v, B} \mid \widehat{L}}{H} .
$$

Proof. Consider two geometric objects which depend on $\omega$ : the translated polyhedral cone $V_{T}^{v, B}+\left(-M_{T}\right)$ with boundary $\partial\left(V_{T}^{v, B}+\left(-M_{T}\right)\right)$, and the line spanned by the vector $H$. Then $V_{T}^{v, B}+\left(-M_{T}\right)$ is generated by $m$ measurable vectors $\xi_{1}, \ldots, \xi_{m}$, where $d \leq m \leq d(d-1)$, and can be represented as the intersection of $l$ half-spaces for some $l$. The $i$ th half-space is spanned by $d-1$ generators $\xi_{i_{1}}, \ldots, \xi_{i_{d-1}}$ from the set $\xi_{1}, \ldots, \xi_{m}$. Putting $g_{i}=\xi_{i_{1}} \times \ldots \times \xi_{i_{d-1}}$, where $\times$ denotes the cross product, we obtain a measurable vector which is orthogonal to each of $\xi_{i_{1}}, \ldots, \xi_{i_{d-1}}$. Thus the $i$ th half-space has the following representation:

$$
\left\{x \in \mathbb{R}^{d}:\left(x-V_{T}^{v, B}\right) \cdot g_{i} \geq 0\right\},
$$


and the boundary of the cone can be represented as follows:

$$
x \in \partial\left(V_{T}^{v, B}+\left(-M_{T}\right)\right) \Leftrightarrow \begin{cases}\left(x-V_{T}^{v, B}\right) \cdot g_{i} \geq 0 \quad \forall i=1, \ldots, l, \\ \left(x-V_{T}^{v, B}\right) \cdot g_{i}=0 & \text { for some } i=1, \ldots, l .\end{cases}
$$

On the other hand the line spanned by the vector $H$ can be represented as

$$
x \in \operatorname{span}\{H\} \Leftrightarrow x \cdot h_{i}=0 \forall i=1, \ldots, d-1,
$$

where $\left\{H, h_{1}, \ldots, h_{d-1}\right\}$ is a basis in $\mathbb{R}^{d}$, each vector $h_{i}$ is measurable and $H \perp h_{i}$ for all $i=1, \ldots, d-1$. Such a basis can be obtained by taking the set $\left\{H, H+e_{1}, \ldots, H+e_{d}\right\}$, where $\left\{e_{1}, \ldots, e_{d}\right\}$ is a standard basis in $\mathbb{R}^{d}$, choosing a subset of $d$ linearly independent vectors containing $H$ and then orthogonalizing it starting from the vector $H$.

There exists exactly one positive point $\widehat{V}$ in the intersection of $\partial\left(V_{T}^{v, B}+\right.$ $\left.\left(-M_{T}\right)\right)$ with $\operatorname{span}\{H\}$. Since it is a solution of a linear system with measurable coefficients,

$$
\begin{cases}\left(x-V_{T}^{v, B}\right) \cdot g_{i} \geq 0 & \forall i=1, \ldots, l \\ \left(x-V_{T}^{v, B}\right) \cdot g_{i}=0 & \text { for some } i=1, \ldots, l \\ x \cdot h_{i}=0 & \forall i=1, \ldots, d-1\end{cases}
$$

it is a measurable random vector. Hence $\widehat{c}$ is also measurable, where $\widehat{V}=\widehat{c} H$. Each transfer is represented by adding to $V_{T}^{v, B}$ some vector from the cone $-M_{T}$. For $\widehat{L}$ we take the transfer represented by $\widehat{V}-V_{T}^{v, B}$. From the construction of $\widehat{V}$ we conclude that for any other proportional transfer such that $V_{T}^{v, B}{ }_{\mid L}=\bar{c} H$ we have $\bar{c} \leq \widehat{c}$. As a consequence,

$$
\widehat{c}=\operatorname{essip}_{L \in \mathfrak{L}\left(V_{T}^{v, B}, H\right)} \frac{V_{T}^{v, B} \mid L}{H}=\frac{V_{T}^{v, B} \mid \widehat{L}}{H}
$$

REMARK 3.5. The success function fulfils

$$
0 \leq \varphi_{v, B} \mathbf{1}_{\left\{V_{T}^{v, B} \succeq_{T} H\right\}^{c}}<1
$$

Proof. We have $\varphi_{v, B} \mathbf{1}_{\left\{V_{T}^{v, B} \succeq_{T} H\right\}^{\mathrm{c}}} \geq 0$ since $(v, B)$ is admissible. If $\varphi_{v, B} \mathbf{1}_{\left\{V_{T}^{v, B} \succeq_{T} H\right\}^{\mathrm{c}}} \geq 1$ then $\mathbf{1}_{\left\{V_{T}^{v, B} \succeq_{T} H\right\}^{\mathrm{c}}}\left(V_{T}^{v, B}{ }_{\mid \widehat{L}} / H\right) \geq 1$. This implies $V_{T}^{v, B} \mid \widehat{L}$ $\geq H$ on $\left\{V_{T}^{v, B} \succeq_{T} H\right\}^{c}$; but this means that $V_{T}^{v, B} \succeq_{T} H$, which is a contradiction.

To summarize, the success function $\varphi_{v, B}$ is equal to 1 if $V_{T}^{v, B} \succeq_{T} H$, and is strictly smaller than 1 if $V_{T}^{v, B} \nsucceq_{T} H$.

In the next part of the paper we will work with the set

$$
\mathcal{R}:=\left\{\varphi: 0 \leq \varphi \leq 1 ; \varphi \text { is } \mathcal{F}_{T} \text {-measurable }\right\}
$$

of $\mathcal{F}_{T}$-measurable functions with values in $[0,1]$. 
We start with two useful properties of the success function.

Lemma 3.6. Assume that $(v, B)$ is an admissible strategy. Then $v \in$ $\Gamma\left(H \varphi_{v, B}\right)$.

Proof. In view of Lemma 3.4 we have

$$
\begin{aligned}
H \varphi_{v, B} & =H 1_{\left\{V_{T}^{v, B} \succeq_{T} H\right\}}+H \operatorname{essup}_{L \in \mathcal{L}\left(V_{T}^{v, B}, H\right)} \frac{V_{T}^{v, B} \mid L}{H} \mathbf{1}_{\left\{V_{T}^{v, B} \succeq_{T} H\right\}^{\mathrm{c}}} \\
& =H \mathbf{1}_{\left\{V_{T}^{v, B} \succeq_{T} H\right\}}+V_{T}^{v, B} \mid \mathbf{L}_{\left\{V_{T}^{v, B} \succeq_{T} H\right\}^{\mathrm{c}} T} \preceq V_{T}^{v, B}
\end{aligned}
$$

where $\widehat{L}$ is an optimal proportional transfer. Thus $v \in \Gamma\left(H \varphi_{v, B}\right)$.

LEMMA 3.7. Assume that $(v, B)$ is a hedging strategy for a modified contingent claim $H \varphi$ for some function $\varphi \in \mathcal{R}$. Then $\varphi_{v, B} \geq \varphi$.

Proof. Since $V_{T}^{v, B} \succeq_{T} H \varphi$, there exists a transfer $M \in L^{0}\left(M_{+}^{d}, \mathcal{F}_{T}\right)$ such that $V_{T}^{v, B}{ }_{\mid M}-H \varphi \geq 0$. Let $N \in \mathfrak{L}\left(V_{T}^{v, B} \mid M-H \varphi, H\right)$ be any proportional transfer on the set $\left\{V_{T}^{v, B} \succeq_{T} H\right\}^{\mathrm{c}}$ such that $\left(V_{T}^{v, B}{ }_{\mid M}-H \varphi\right)_{\mid N} / H=\gamma$ for some $\gamma \geq 0$. Consider the terminal wealth $V_{T}^{v, B}$ on the set $\left\{V_{T}^{v, B} \succeq_{T} H\right\}^{\mathrm{c}}$ after transfer $K$ described as follows: first change $V_{T}^{v, B}$ by transfer $M$ and then change $V_{T}^{v, B}{ }_{\mid M}-H \varphi$ by transfer $N$. The terminal wealth $V_{T}^{v, B}$ after transfer $K$ is

$$
V_{T}^{v, B}{ }_{\mid K}=H \varphi+\left(V_{T}^{v, B}{ }_{\mid M}-H \varphi\right)_{\mid N} .
$$

It is clear that $K \in \mathfrak{L}\left(V_{T}^{v, B}, H\right)$ since

$$
V_{T}^{v, B}{ }_{\mid K}=H \varphi+H \gamma=(\varphi+\gamma) H .
$$

This leads to the following inequalities:

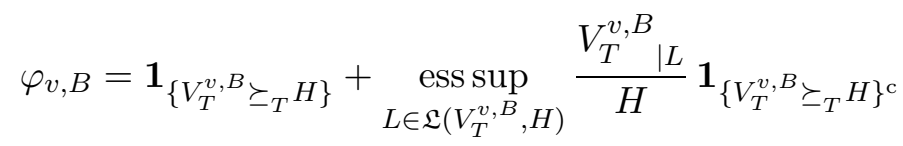

$$
\begin{aligned}
& \geq \mathbf{1}_{\left\{V_{T}^{v, B} \succeq_{T} H\right\}}+\frac{V_{T}^{v, B} \mid K}{H} \mathbf{1}_{\left\{V_{T}^{v, B} \succeq_{T} H\right\}^{\mathrm{c}}} \\
& =\mathbf{1}_{\left\{V_{T}^{v, B} \succeq_{T} H\right\}}+(\varphi+\gamma) \mathbf{1}_{\left\{V_{T}^{v, B} \succeq_{T} H\right\}^{c}} \geq \varphi .
\end{aligned}
$$

4. Quantile hedging - effectiveness maximization. The set $\Gamma(H)$ is the set of all initial endowments which allow one to hedge the contingent claim $H$. If $v \in \Gamma(H)$ then there exists a strategy $B \in \mathcal{B}$ such that $V_{T}^{v, B} \succeq_{T} H$. Suppose that we are given an initial capital $v_{0}$ such that $v_{0} \notin \Gamma(H)$. A natural question arises: what is an optimal strategy for $v_{0}$ ? As optimality criterion we take the expectation of the success function under 
measure $P$. If $\mathbf{E}\left[\varphi_{v, B}\right] \geq \mathbf{E}\left[\varphi_{\bar{v}, \bar{B}}\right]$ for two admissible strategies $(v, B)$ and $(\bar{v}, \bar{B})$ then the strategy $(v, B)$ is at least as effective as $(\bar{v}, \bar{B})$. If $(v, B)$ is at least as effective as any other admissible strategy, then it is called optimal. The problem of finding an optimal strategy for $v_{0}$ is the first aspect of the quantile hedging problem and we formally formulate it as follows:

For a fixed initial endowment $v_{0} \in \Gamma(0)$ such that $v_{0} \notin \Gamma(H)$ find an admissible strategy $(v, B)$, where $v_{0} \succeq_{0} v$, such that $\mathbf{E}\left[\varphi_{v, B}\right] \rightarrow \max$.

To describe an optimal strategy, we start with the following theorem.

THEOREM 4.1. There exists a function $\widetilde{\varphi} \in \mathcal{R}$ which is a solution of the problem

$$
\mathbf{E}[\varphi] \rightarrow \max , \quad v_{0} \in \Gamma(H \varphi) .
$$

Proof. Set $\mathcal{R}_{0}:=\left\{\varphi \in \mathcal{R}: v_{0} \in \Gamma(H \varphi)\right\}$. Then $\mathcal{R}_{0} \neq \emptyset$ since $0 \in \mathcal{R}_{0}$. Let $\varphi_{n} \in \mathcal{R}_{0}$ be a sequence such that $\mathbf{E}\left[\varphi_{n}\right] \rightarrow \sup _{\varphi \in \mathcal{R}_{0}} \mathbf{E}[\varphi]$. Since $\left\{\varphi_{n}\right\}$ is a sequence of elements from a hull in $L^{\infty}(\Omega)$, there exists a subsequence $\varphi_{n_{k}}$ which converges to some $\widetilde{\varphi}$ in the weak $*$ topology. One can prove that $\widetilde{\varphi}$ belongs to $\mathcal{R}$. We will show that $v_{0} \in \Gamma(H \widetilde{\varphi})$. Each $\varphi_{n}$ satisfies $\widehat{Z}_{0} v_{0} \geq$ $\mathbf{E}\left[\widehat{Z}_{T} H \varphi_{n}\right]$ for all $Z \in \mathcal{Z}$, and $\widetilde{\varphi}$ as a weak limit satisfies

$$
\forall Z \in \mathcal{Z} \quad \widehat{Z}_{0} v_{0} \geq \mathbf{E}\left[\widehat{Z}_{T} H \varphi_{n_{k}}\right] \underset{k}{\rightarrow} \mathbf{E}\left[\widehat{Z}_{T} H \widetilde{\varphi}\right] .
$$

Thus $v_{0} \in \Gamma(H \widetilde{\varphi})$.

The next theorem provides the solution of our problem.

THEOREM 4.2. Let $\widetilde{\varphi}$ be the function from Theorem 4.1, and let $\left(v_{0}, B\right)$ be a hedging strategy for the modified contingent claim $H \widetilde{\varphi}$. Then $\left(v_{0}, B\right)$ is an optimal strategy. Furthermore, $\widetilde{\varphi}=\varphi_{v_{0}, B}$.

Proof. The strategy $\left(v_{0}, B\right)$ is admissible since $V_{T}^{v_{0}, B} \succeq_{T} H \widetilde{\varphi} \succeq_{T}$. Let $(\bar{v}, \bar{B})$ be any admissible strategy such that $v_{0} \succeq_{0} \bar{v}$. Then $\bar{v} \in \Gamma\left(H \varphi_{\bar{v}, \bar{B}}\right)$ by Lemma 3.6, and this implies that $v_{0} \in \Gamma\left(H \varphi_{\bar{v}, \bar{B}}\right)$. From Theorem 4.1 we have

$$
\mathbf{E}\left[\varphi_{\bar{v}, \bar{B}}\right] \leq \mathbf{E}[\widetilde{\varphi}] .
$$

Now, consider the strategy $\left(v_{0}, B\right)$. Since $V_{T}^{v_{0}, B} \succeq_{T} H \widetilde{\varphi}$, by Lemma 3.7 we have

$$
\varphi_{v_{0}, B} \geq \widetilde{\varphi}
$$

By (4.2.1) and (4.2.2) we have $\varphi_{v_{0}, B}=\widetilde{\varphi}$. Hence $\left(v_{0}, B\right)$ is optimal.

5. Quantile hedging - sets with a fixed level of effectiveness. Given $\varepsilon \in[0,1]$, we want to characterize the strategies with effectiveness 
not smaller than $1-\varepsilon$. This is the second aspect of quantile hedging, and in fact our task is to characterize the set

$\Gamma_{\varepsilon}(H)=\left\{v \in \mathbb{R}^{d}:\right.$ there exists an admissible strategy $B$ such that $\left.\mathbf{E}\left[\varphi_{v, B}\right] \geq 1-\varepsilon\right\}$.

It is clear that $\Gamma_{\varepsilon_{1}}(H) \subseteq \Gamma_{\varepsilon_{2}}(H)$ if $\varepsilon_{1} \leq \varepsilon_{2}$. Hence $\Gamma_{\varepsilon}(H)$ contains $\Gamma(H)=$ $\Gamma_{0}(H)$ for any $\varepsilon \in[0,1]$, but it can contain other elements which are initial capitals allowing one to hedge $H$ with some loss of effectiveness.

Set

$$
\mathcal{M}:=\{\varphi \in \mathcal{R}: \mathbf{E}[\varphi] \geq 1-\varepsilon\} .
$$

The next theorem provides a description of the set $\Gamma_{\varepsilon}(H)$.

THEOREM 5.1. The set $\Gamma_{\varepsilon}(H)$ admits the following representation:

$$
\Gamma_{\varepsilon}(H)=\bigcup_{\varphi \in \mathcal{M}} \Gamma(H \varphi) .
$$

Proof. $\subseteq$ : Let $v \in \Gamma_{\varepsilon}(H)$. Then there exists $B \in \mathcal{B}$ such that $V_{T}^{v, B} \succeq_{T} 0$ and $\mathbf{E}\left[\varphi_{v, B}\right] \geq 1-\varepsilon$. Thus $\varphi_{v, B} \in \mathcal{M}$ and

$$
\Gamma\left(H \varphi_{v, B}\right) \subseteq \bigcup_{\varphi \in \mathcal{M}} \Gamma(H \varphi) .
$$

But $v \in \Gamma\left(H \varphi_{v, B}\right)$ by Lemma 3.6, and thus $v \in \bigcup_{\varphi \in \mathcal{M}} \Gamma(H \varphi)$.

$\supseteq$ : Let $v \in \bigcup_{\varphi \in \mathcal{M}} \Gamma(H \varphi)$. Then there exists $\varphi \in \mathcal{M}$ such that $v \in$ $\Gamma(H \varphi)$. Consider a strategy $(v, B)$ which hedges the modified contingent claim $H \varphi$. Then by Lemma 3.7 we have

$$
V_{T}^{v, B} \succeq_{T} H \varphi \Rightarrow \varphi_{v, B} \geq \varphi
$$

and so $\mathbf{E}\left[\varphi_{v, B}\right] \geq \mathbf{E}[\varphi] \geq 1-\varepsilon$, that is, $v \in \Gamma_{\varepsilon}(H)$.

6. Risk measure in quantile hedging - minimizing shortfall risk. On markets without transaction costs the shortfall is defined as $\left(C-X_{T}^{x, B}\right)^{+}$, where $a^{+}=\max \{a, 0\}$. In this section we introduce a shortfall connected with the strategy $(v, B)$ under transaction costs. To this end we use the set of proportional transfers. The shortfall risk is introduced as the expectation of a loss function of shortfall. Our aim is to minimize the shortfall risk for a fixed initial capital over all admissible strategies.

In Section 3 we introduced the random variable

$$
\operatorname{ess~sup}_{L \in \mathfrak{L}\left(V_{T}^{v, B}, H\right)} V_{T}^{v, B}{ }_{\mid L} / H
$$

defined on the set $\left\{V_{T}^{v, B} \succeq_{T} H\right\}^{\mathrm{c}}$. It describes the part of the contingent claim which is successfully hedged. For shortfall, we take the remaining part: $1-\operatorname{ess} \sup _{L \in \mathfrak{L}\left(V_{T}^{v, B}, H\right)} V_{T}^{v, B}{ }_{\mid L} / H$. Let us start with the formal definition. 
Definition 6.1. The shortfall of an admissible strategy $(v, B)$ is the random variable defined by

$$
s\left(V_{T}^{v, B}\right)= \begin{cases}0 & \text { on }\left\{V_{T}^{v, B} \succeq_{T} H\right\}, \\ 1-\operatorname{ess~sup~}_{L \in \mathfrak{L}\left(V_{T}^{v, B}, H\right)} \frac{V_{T}^{v, B} \mid L}{H} & \text { on }\left\{V_{T}^{v, B} \succeq_{T} H\right\}^{\mathrm{c}} .\end{cases}
$$

REMARK 6.2. The shortfall can be expressed in terms of the success function:

$$
\begin{aligned}
1-\varphi_{v, B} & =0 \cdot \mathbf{1}_{\left\{V_{T}^{v, B} \succeq_{T} H\right\}}+\left(1-\operatorname{ess~sup}_{L \in \mathfrak{L}\left(V_{T}^{v, B}, H\right)} \frac{V_{T}^{v, B} \mid L}{H}\right) \mathbf{1}_{\left\{V_{T}^{v, B} \succeq_{T} H\right\}^{\mathrm{c}}} \\
& =s\left(V_{T}^{v, B}\right) .
\end{aligned}
$$

The shortfall is a random variable with values in $[0,1]$. It is equal to 0 if $V_{T}^{v, B} \succeq_{T} H$ and it is strictly positive if $V_{T}^{v, B} \nsucceq_{T} H$.

Let $u:[0,1] \rightarrow \mathbb{R}$ be a continuous, non-decreasing function such that $u(0)=0$ and $u(1)<\infty$. We regard such a function as a loss function. Basing on a loss function we define the shortfall risk of an admissible strategy as $\mathbf{E}\left[u\left(s\left(V_{T}^{v, B}\right)\right)\right]$. It is clear that if $v \in \Gamma(H)$ then the shortfall risk for a hedging strategy is equal to 0 , otherwise it is positive. If $\mathbf{E}\left[u\left(s\left(V_{T}^{v, B}\right)\right)\right] \leq$ $\mathbf{E}\left[u\left(s\left(V_{T}^{\bar{v}, \bar{B}}\right)\right)\right]$ for two admissible strategies $(v, B)$ and $(\bar{v}, \bar{B})$ then we regard the strategy $(v, B)$ as not as risky as $(\bar{v}, \bar{B})$. If the shortfall risk of the strategy $(v, B)$ is not greater than any other, then $(v, B)$ is called optimal or risk-minimizing.

Similarly to the previous sections we formulate the first aspect of the risk measure problem as:

For a fixed initial endowment $v_{0} \in \Gamma(0)$ such that $v_{0} \notin \Gamma(H)$ find an admissible strategy $(v, B)$, where $v_{0} \succeq_{0} v$, such that

$$
\mathbf{E}\left[u\left(s\left(V_{T}^{v, B}\right)\right)\right] \rightarrow \min .
$$

We start with an auxiliary lemma proved in [3].

Lemma 6.3. Let $X_{1}, X_{2}, \ldots$ be a sequence of $[0, \infty)$-valued random variables. There exists a sequence $\widetilde{X}_{n} \in \operatorname{conv}\left\{X_{n}, X_{n+1}, \ldots\right\}$ such that $\widetilde{X}_{n}$ converges almost surely to a $[0, \infty]$-valued random variable $\widetilde{X}$.

To describe an optimal strategy we start with the following theorem.

THEOREM 6.4. There exists a function $\widetilde{\varphi} \in \mathcal{R}$ which is a solution of the problem

$$
\mathbf{E}[u(1-\varphi)] \rightarrow \min , \quad v_{0} \in \Gamma(H \varphi) .
$$


Proof. Set $\mathcal{R}_{0}:=\left\{\varphi \in \mathcal{R}: v_{0} \in \Gamma(H \varphi)\right\}$. Then $\mathcal{R}_{0} \neq \emptyset$ since $0 \in \mathcal{R}_{0}$. Let $\varphi_{n} \in \mathcal{R}_{0}$ be a sequence such that $\mathbf{E}\left[u\left(1-\varphi_{n}\right)\right] \rightarrow \inf _{\varphi \in \mathcal{R}_{0}} \mathbf{E}[u(1-\varphi)]$. In view of Lemma 6.3 there exists a sequence $\widetilde{\varphi}_{n} \in \operatorname{conv}\left\{\varphi_{n}, \varphi_{n+1}, \ldots\right\}$ which converges almost surely to $\widetilde{\varphi} \in \mathcal{R}$. Since $u\left(1-\widetilde{\varphi}_{n}\right) \leq u(1)<\infty$, by the dominated convergence theorem we obtain

$$
\mathbf{E}[u(1-\widetilde{\varphi})]=\lim _{n \rightarrow \infty} \mathbf{E}\left[u\left(1-\widetilde{\varphi}_{n}\right)\right]=\inf _{\varphi \in \mathcal{R}_{0}} \mathbf{E}[u(1-\varphi)] .
$$

From Fatou's lemma we have

$$
\mathbf{E}\left[\widehat{Z}_{T} H \widetilde{\varphi}\right]=\mathbf{E}\left[\lim _{n} \widehat{Z}_{T} H \widetilde{\varphi}_{n}\right] \leq \liminf _{n} \mathbf{E}\left[\widehat{Z}_{T} H \widetilde{\varphi}_{n}\right] \leq \widehat{Z}_{0} v_{0} \quad \forall Z \in \mathcal{Z} .
$$

Hence $v_{0} \in \Gamma(H \widetilde{\varphi})$.

The next theorem provides a description of a risk-minimizing strategy for $v_{0}$.

THEOREM 6.5. Let $\widetilde{\varphi}$ be the function from Theorem 6.4 , and let $\left(v_{0}, B\right)$ be a hedging strategy for the modified contingent claim $H \widetilde{\varphi}$. Then $\left(v_{0}, B\right)$ is an optimal strategy. Furthermore, $\widetilde{\varphi}=\varphi_{v_{0}, B}$.

Proof. The strategy $\left(v_{0}, B\right)$ is admissible since $V_{T}^{v_{0}, B} \succeq_{T} H \widetilde{\varphi} \succeq_{T}$ 0. Let $(\bar{v}, \bar{B})$ be any admissible strategy such that $v_{0} \succeq_{0} \bar{v}$. Then $\bar{v} \in \Gamma\left(H \varphi_{\bar{v}, \bar{B}}\right)$ by Lemma 3.6, which implies $v_{0} \in \Gamma\left(H \varphi_{\bar{v}, \bar{B}}\right)$. From Remark 6.2 and Theorem 6.4 we obtain

$$
\mathbf{E}\left[u\left(s\left(V_{T}^{\bar{v}, \bar{B}}\right)\right)\right]=\mathbf{E}\left[u\left(1-\varphi_{\bar{v}, \bar{B}}\right)\right] \geq \mathbf{E}[u(1-\widetilde{\varphi})] .
$$

Now consider the strategy $\left(v_{0}, B\right)$. Since $V_{T}^{v_{0}, B} \succeq_{T} H \widetilde{\varphi}$, by Lemma 3.7 we have

$$
\varphi_{v_{0}, B} \geq \widetilde{\varphi}
$$

Taking (6.5.3) and (6.5.4) into account we have $\varphi_{v_{0}, B}=\widetilde{\varphi}$, so $\mathbf{E}\left[u\left(s\left(V_{T}^{v_{0}, B}\right)\right)\right]$ $=\mathbf{E}[u(H-H \widetilde{\varphi})]$, and this proves that $\left(v_{0}, B\right)$ is optimal.

7. Risk measure in quantile hedging - sets with a fixed level of shortfall risk. Given $\alpha \geq 0$, we want to characterize the strategies with shortfall risk not greater than $\alpha$. This is the second aspect of the risk measure problem in quantile hedging. Our task is to provide a description of the set

$$
\Gamma_{\alpha}^{u}(H):=\left\{v \in \mathbb{R}^{d}: \text { there exists an admissible strategy } B\right.
$$

$$
\text { such that } \left.\mathbf{E}\left[u\left(s\left(V_{T}^{v, B}\right)\right)\right] \leq \alpha\right\} \text {. }
$$

It is clear that $\Gamma_{\alpha_{1}}^{u}(H) \subseteq \Gamma_{\alpha_{2}}^{u}(H)$ if $\alpha_{1} \leq \alpha_{2}$. Since $\mathbf{E}\left[u\left(s\left(V_{T}^{v, B}\right)\right)\right]=0$ for the hedging strategy $(v, B)$, we conclude that $\Gamma_{\alpha}^{u}(H)$ contains $\Gamma(H)=\Gamma_{0}^{u}(H)$ for any $\alpha \geq 0$. 
Set

$$
\mathcal{N}:=\{\varphi \in \mathcal{R}: \mathbf{E}[u(1-\varphi)] \leq \alpha\} .
$$

The next theorem provides a description of the set $\Gamma_{\alpha}^{u}(H)$.

THEOREM 7.1. The set $\Gamma_{\alpha}^{u}(H)$ admits the following representation:

$$
\Gamma_{\alpha}^{u}(H)=\bigcup_{\varphi \in \mathcal{N}} \Gamma(H \varphi)
$$

Proof. The proof is similar to the proof of Theorem 5.1.

$\subseteq$ : Let $v \in \Gamma_{\alpha}^{u}(H)$. Then there exists $B \in \mathcal{B}$ such that $V_{T}^{v, B} \succeq_{T} 0$ and $\mathbf{E}\left[u\left(s\left(V_{T}^{v, B}\right)\right)\right]=\mathbf{E}\left[u\left(1-\varphi_{v, B}\right)\right] \leq \alpha$. Thus $\varphi_{v, B} \in \mathcal{N}$ and by Lemma 3.6 we have

$$
v \in \Gamma\left(H \varphi_{v, B}\right) \subseteq \bigcup_{\varphi \in \mathcal{N}} \Gamma(H \varphi) .
$$

?: Let $v \in \bigcup_{\varphi \in \mathcal{N}} \Gamma(H \varphi)$. Then there exists $\varphi \in \mathcal{N}$ such that $v \in$ $\Gamma(H \varphi)$. Consider a strategy $(v, B)$ which hedges the modified contingent claim $H \varphi$. Then by Lemma 3.7 we have

$$
V_{T}^{v, B} \succeq_{T} H \varphi \Rightarrow \varphi_{v, B} \geq \varphi
$$

and this implies

$$
\mathbf{E}\left[u\left(s\left(V_{T}^{v, B}\right)\right)\right]=\mathbf{E}\left[u\left(1-\varphi_{v, B}\right)\right] \leq \mathbf{E}[u(1-\varphi)] \leq \alpha,
$$

so that $v \in \Gamma_{\alpha}^{u}(H)$.

8. Quantile hedging under zero transaction costs. In this section we show how the theory of Föllmer and Leukert can be obtained. All previous sections required the EF condition, which of course is not satisfied under zero transaction costs. We will base on the results obtained by Delbaen, Kabanov and Valkeila [2], which are less general than the results used so far, but the condition $\mathbf{E F}$ is not required. First, we give a short description of these results, then recall the two aspects of quantile hedging studied by Föllmer and Leukert, and finally show how their theory can be obtained under zero transaction costs.

In [2] it is assumed that the transaction costs are constant in time, given by a matrix $\Lambda$. The contingent claim is bounded from below in the sense of the partial ordering determined by the cone $K:=M+\mathbb{R}_{+}^{d}$, that is, $H \succeq c \mathbf{1}$ for some $c \in \mathbb{R} ; K$ is independent of $t$ and $\omega$. We denote by $\mathcal{Q}$ the set of probability measures $Q \sim P$ such that $S_{t}$ follows a local martingale with respect to $Q$. We shall need the condition

$$
\text { EMM : } \quad \mathcal{Q} \neq \emptyset .
$$

Let $\mathcal{D}$ be the set of martingales $Z$ with $\widehat{Z}$ taking values in $K^{*}$ and with $\widehat{Z}_{T}$ bounded. Under EMM we have the following description of the set of 
hedging endowments:

$$
\Gamma(H)=\bigcap_{Z \in \mathcal{D}}\left\{v \in \mathbb{R}^{d}: \widehat{Z}_{0} v \geq \mathbf{E} \widehat{Z}_{T} H\right\} .
$$

It is left as an exercise to check that under this new description of $\Gamma(H)$ Theorems 4.1, 4.2, 5.1, which solve our problems, remain true.

Now consider a classical market model without transaction costs. Under the no-arbitrage condition the price of a scalar contingent claim $C$ is given as $\sup _{Q \in \mathcal{Q}} \mathbf{E}^{Q}[C]$. In the quantile hedging problem studied by Föllmer and Leukert only those admissible strategies $(x, B)$ are considered for which the wealth process $X_{t}^{x, B}$ is non-negative for all $t=0,1, \ldots, T$. The authors use as an effectiveness measure the success function defined as

$$
\varphi_{x, B}=\mathbf{1}_{\left\{X_{T}^{x, B} \geq C\right\}}+\frac{X_{T}^{x, B}}{C} \mathbf{1}_{\left\{X_{N}^{x, B}<C\right\}} .
$$

THE FIRST PROBLEM

Let $x_{0}<\sup _{Q \in \mathcal{Q}} \mathbf{E}^{Q}[C]$ be a fixed initial endowment. We search for an admissible strategy $(x, B)$, where $x \leq x_{0}$, maximizing $\mathbf{E}\left[\varphi_{x, B}\right]$. We write this problem as

$$
\mathbf{E}\left[\varphi_{x, B}\right] \rightarrow \max , \quad x \leq x_{0} .
$$

THE SECOND PROBLEM

Let $\varepsilon$ be a fixed number in $[0,1]$. We search for an admissible strategy $(x, B)$ with effectiveness not smaller than $1-\varepsilon$ and minimizing the initial capital. We write this problem as

$$
\mathbf{E}\left[\varphi_{x, B}\right] \geq 1-\varepsilon, \quad x \rightarrow \min .
$$

To show that these problems can be formulated under zero transaction costs we have to find scalar equivalents of multi-dimensional objects on our market. Let $Y \in \mathbb{R}^{d}$ describe how our wealth is allocated in stock positions on the market with transaction costs. Now choose the $i$ th stock account to transfer capitals from all others to it. Then the wealth of $Y$ in the $i$ th stock is

$$
Y(i):=\sum_{j=0}^{d}\left(1-\lambda^{j i}\right) Y^{j} .
$$

Usually $Y(i) \neq Y(j)$ for $i \neq j$, but under zero transaction costs we have $Y(i)=Y(j)=\sum_{i=1}^{d} Y^{i}$. Thus we adopt the following scalar equivalents: for the initial endowment $v$ we take $x_{v}:=\sum v^{i}$, for the wealth process $V_{t}^{v, B}$ we take $X_{t}^{x_{v}, B}:=\sum\left(V_{t}^{v, B}\right)^{i}$, for the contingent claim $H$ we take $C_{H}:=\sum H^{i}$.

Now we show that the problems of quantile hedging under zero transaction costs are the same as those formulated in [2] for scalar equivalents. 
First, note that

$$
\frac{V_{T}^{v, B} \mid L}{H}=\frac{\sum\left(V_{T}^{v, B}\right)^{i}}{\sum H^{i}} \quad \forall L \in \mathfrak{L}\left(V_{T}, H\right) .
$$

For each $L \in \mathfrak{L}\left(V_{T}, H\right)$ we have

$$
\sum \frac{V_{T}^{v, B} \mid L}{H} H^{i}=\frac{V_{T}^{v, B} \mid L}{H} \sum H^{i} \quad \text { and } \quad \sum \frac{V_{T}^{v, B} \mid L}{H} H^{i}=\sum\left(V_{T}^{v, B} \mid L\right)^{i},
$$

so

$$
\frac{V_{T}^{v, B} \mid L}{H}=\frac{\sum\left(V_{T}^{v, B} \mid L\right)^{i}}{\sum H^{i}}
$$

Since the costs are equal to zero, we have $\sum\left(V_{T}^{v, B}{ }_{\mid L}\right)^{i}=\sum\left(V_{T}^{v, B}\right)^{i}$ and (8.0.5) holds.

Since the relation " $\succeq$ " becomes the linear ordering " $\geq$ " for the sums of components, we get the equality of the success functions:

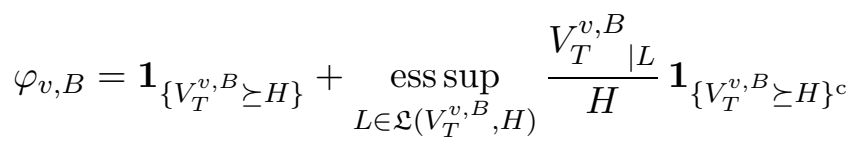

$$
\begin{aligned}
& =\mathbf{1}_{\left\{\sum\left(V_{T}^{v, B}\right)^{i} \geq \sum H^{i}\right\}}+\frac{\sum\left(V_{T}^{v, B}\right)^{i}}{\sum H^{i}} \mathbf{1}_{\left\{\sum\left(V_{T}^{v, B}\right)^{i}<\sum H^{i}\right\}} \\
& =\mathbf{1}_{\left\{X_{T}^{x_{v}, B} \geq C_{H}\right\}}+\frac{X_{T}^{x_{v}, B}}{C_{H}} \mathbf{1}_{\left\{X_{T}^{x_{v}, B}<C_{H}\right\}}=\varphi_{x_{v}, B} .
\end{aligned}
$$

One can check that the set of hedging endowments is of the form $\Gamma(H)=$ $\left\{v \in \mathbb{R}^{d}: \sum v^{i} \geq \sup _{Q \in \mathcal{Q}} \mathbf{E}^{Q}\left[\sum H^{i}\right]\right\}$. Thus our problem of maximizing the effectiveness,

$$
\mathbf{E}\left[\varphi_{v, B}\right] \rightarrow \max , \quad v \preceq v_{0} \notin \Gamma(H)
$$

becomes

$$
\mathbf{E}\left[\varphi_{x, B}\right] \rightarrow \max , \quad x \leq x_{v_{0}}<\sup _{Q \in \mathcal{Q}} \mathbf{E}^{Q}\left[C_{H}\right],
$$

which is the first problem considered by Föllmer and Leukert.

Our second problem is to determine the set $\Gamma_{\varepsilon}(H)$. First note that if for $v, \bar{v} \in \mathbb{R}^{d}$ we have $\sum \bar{v}^{i} \geq \sum v^{i}$ and $v \in \Gamma_{\varepsilon}(H)$ then $\bar{v} \in \Gamma_{\varepsilon}(H)$. For $\gamma_{v}:=$ $\sum v^{i}$ define $\gamma:=\inf _{v \in \Gamma_{\varepsilon}(H)} \gamma_{v}$. If $\sum v^{i} \geq \gamma$ for some $v \in \mathbb{R}^{d}$ then $v \in \Gamma_{\varepsilon}(H)$, and if $\sum v^{i}<\gamma$ then $v \notin \Gamma_{\varepsilon}(H)$. Thus $\Gamma_{\varepsilon}(H)=\left\{v \in \mathbb{R}^{d}: \sum v^{i} \geq \gamma\right\}$. The problem reduces to finding the number $\gamma$, which is the cost minimizing capital searched by Föllmer and Leukert.

REMARK 8.1. Föllmer and Leukert considered admissible strategies for which $X_{t}^{x, B} \geq 0$ for each $t=0,1, \ldots, T$. We only require $X_{T}^{x_{v}, B} \geq 0$, which is a generalization. 
Acknowledgements. I would like to thank Professor Łukasz Stettner for helpful discussions and Ania Stasiuk for her help with editing this paper.

\section{References}

[1] J. Cvitanić and I. Karatzas, On dynamic measures of risk, Finance Stoch. 3 (1999), 451-482.

[2] F. Delbaen, Yu. M. Kabanov and E. Valkeila, Hedging under transaction costs in currency markets: a discrete-time model, Math. Finance 12 (2002), 45-61.

[3] F. Delbaen and W. Schachermayer, A general version of the fundamental theorem of asset pricing, Math. Ann. 300 (1994), 463-520.

[4] H. Föllmer and P. Leukert, Quantile hedging, Finance Stoch. 3 (1999), 251-273.

[5] -, 一, Efficient hedging: cost versus shortfall risk, ibid. 4 (2000), 117-146.

[6] Yu. M. Kabanov, M. Rásonyi and C. Stricker, No-arbitrage criteria for financial markets with efficient friction, ibid., to appear.

[7] Yu. M. Kabanov and C. Stricker, The Harrison-Pliska arbitrage pricing theorem under transaction costs, J. Math. Econom. 35 (2001), 185-196.

[8] H. Pham, Dynamic $L^{p}$-hedging in discrete time under cone constrains, SIAM J. Control Optim. 38 (2000), 665-682.

Institute of Mathematics

Polish Academy of Sciences

Śniadeckich 8

00-956 Warszawa, Poland

E-mail: mb@impan.gov.pl

Received on 20.6.2002;

revised version on 10.10.2002 